\title{
Gestión de cuencas y turismo. Caso de la cuenca del Río Frío, Guatuso, Costa Rica
}

\section{Basin management and tourism. Frio River case study, Guatuso, Costa Rica}

\author{
FÉlix Zumbado-Morales* \\ Roger MesÉn-LeaL*
}

\begin{abstract}
In 2014, the National System of Conservation Areas (SINAC) of Costa Rica bid the project for the Tourism Development Plan of the County of Guatuso and the district of Curubandé in Liberia, Guanacaste. Project funds came from the Inter-American Development Bank (IDB) through the IDB-Tourism project. The Sustainable Urban Development Research Program (ProDUS) of the University of Costa Rica won the tender and carried out the project. As part of the results, ProDUS-UCR found out the problem of lack of planning in the "Rio Frio" basin and the consequent negative impact on the tourism sector.
\end{abstract}

Keywords: basin management, tourism system, Guatuso, environmental management.

\section{Resumen}

En el 2014, el Sistema Nacional de Áreas de Conservación (SINAC) de Costa Rica licitó el proyecto Plan de Desarrollo Turístico del Cantón de Guatuso y el distrito de Curubandé en Liberia, Guanacaste, con fondos del Banco Interamericano de Desarrollo (BID). El Programa de Investigación en Desarrollo Urbano Sostenible (ProDUS) de la Universidad de Costa Rica ganó la licitación y realizó el proyecto. Este artículo pretende mostrar cómo se ve afectado el desarrollo del sector turismo por la falta de planificación a nivel de cuenca, utilizando el estudio de caso de la cuenca del Río Frío.

Palabras clave: manejo de cuencas, sistema turístico, Guatuso, gestión ambiental. 


\section{Introducción}

El cantón de Guatuso se ubica en la provincia de Alajuela en las coordenadas geográficas $10^{\circ} 42^{\prime} 22^{\prime \prime}$ latitud norte y $84^{\circ} 49^{\prime} 57^{\prime \prime}$ longitud oeste; tiene una superficie de $759.76 \mathrm{~km}^{2}$ y está conformado por los distritos de San Rafael, Buena Vista y Cote, donde la cabecera del cantón corresponde al poblado de San Rafael.

Se encuentra delimitado por los cantones de Upala, Los Chiles, San Carlos, Tilarán y Cańas. Algunos de los principales ríos que atraviesan el cantón de Guatuso son: Frío, Buena Vista, Rito, Samen, Caño Ciego, Cote y Pejibaye. Para el 2011 el cantón de Guatuso contaba con una población de 15,508 habitantes según el Instituto Nacional de Estadística y Censos (INEC); (ProDUS-UCR, 2015).

En el cantón de Guatuso se encuentran ocho zonas de vida con sus respectivas zonas de transición, entre las que están: bosque húmedo tropical, bosque húmedo tropical transición a perhúmedo, bosque muy húmedo premontano, bosque muy húmedo premontano transición a basal, bosque muy húmedo tropical, bosque muy húmedo tropical transición a premontano, bosque pluvial montano bajo y bosque pluvial premontano (ProDUS-UCR, 2015).

La cobertura de bosque en Guatuso es de menos de 50\% de la superficie total del cantón, la cual se concentra principalmente en el volcán Tenorio y el humedal Cańo Negro.

\section{1. Áreas protegidas}

Las Áreas Silvestres Protegidas (ASP) en Costa Rica se encuentran bajo la administración del Sistema Nacional de Áreas de Conservación (SINAC), el cual se divide en 11 áreas. El cantón de Guatuso se encuentra en la de Conservación Huetar Norte, que está conformada por los parques nacionales: Arenal y Juan Castro Blanco; dos Refugios Nacionales de Vida Silvestre: el Corredor Fronterizo y Las Camelias; dos Refugios Nacionales de Vida Silvestre Mixto: Caño Negro y Maquenque; y un Refugio de Vida Silvestre: Duarú, cuya administración es privada.

En el cantón se ubican dos áreas silvestres protegidas estatales, el Parque Nacional Volcán Tenorio (PNVT), reconocido internacionalmente por la catarata de río Celeste y parte del Humedal Nacional de Vida Silvestre Mixto Caño Negro. El PNVT presentó, al año 2014, una visita de 31,540 turistas (14,473 no residentes y 17,067 residentes). Este parque fue creado el 27 de abril de 1995, cuenta con una extensión de 18,402 hectáreas, de los cuales 12,871 corresponden al área del volcán y 5531 
hectáreas a la zona protectora del Tenorio. Geográficamente se encuentra entre los cantones de Guatuso, Upala, Cañas, Bagaces y Tilarán (SINAC, 2014a).

El Refugio de Vida Silvestre Mixto Caño Negro fue creado el 4 de enero de 1984, tiene una extensión de 9969 hectáreas y se ubica en la sección baja de la cuenca del Río Frío, entre los cantones de Guatuso y los Chiles, $17 \%$ del total de su extensión se encuentra dentro del cantón de Guatuso. La zona presenta un clima tropical lluvioso, dichas lluvias se concentran de mayo a enero con una precipitación anual promedio entre 2500 y $3000 \mathrm{~mm}$. La temperatura en el lugar oscila entre los 25 y los $27^{\circ}$ centígrados (SINAC, 2014b).

Durante la fase del diagnóstico se determinó que uno de los principales problemas del cantón es la deforestación de la parte alta de la cuenca del Río Frío, lo que ha provocado el arrastre de sedimentos hasta el cauce de río; debido a este problema se perdió la mayor parte de la navegabilidad del río y muchos de los sedimentos han sido arrastrados hasta el Refugio de Vida Silvestre Mixto (RNVSM) de Caño Negro.

\section{Cuencas y su gestión}

Una cuenca es la superficie de terreno circundante a un cuerpo de agua superficial definido por divisorias topográficas donde la precipitación que cae sobre el terreno escurre hacia el cuerpo de agua (Comcure, 2010). Según ProDUS-UCR:

el manejo adecuado de una cuenca trae como consecuencia una serie de beneficios al ambiente, a la población y en general al país, cuando se hace de forma integral. La administración debe ser a lo largo de toda la cuenca puesto que las decisiones que se tomen en la parte alta de la cuenca siempre van afectar a las partes bajas de la misma. Es deseable que las decisiones relativas a la cuenca sean coordinadas en una única dirección, ya que de un manejo adecuado de la misma se pueden obtener importantes ventajas, algunas de ellas pueden ser:

- Valorización de la tierra

- Conservación de las propiedades del suelo

- Mejoramiento de los rendimientos productivos agrícolas, lo cual aumenta las ganancias económicas de los productores.

- Garantía de suministro de agua al protegerse las nacientes y sus alrededores.

- Disminución de los procesos de erosión y sedimentación.

- Desarrollo de actividades productivas compatibles con el ambiente, lo cual aumenta el empleo y los ingresos de la población.

- Mejoramiento de la calidad de vida de los habitantes (ProDUS-UCR, 2015: 72) 


\section{La Secretaría de la Convención de Ramsar}

... establece que el objetivo del manejo de las cuencas hidrográficas debería ser armonizar las estrategias sobre los recursos hídricos con las relativas al uso de la tierra, de manera que se puedan aplicar conjuntamente, a fin de contribuir a la conservación de humedales sanos y funcionales que brinden una gama completa de servicios a las personas (incluido el abastecimiento de agua). Un proceso claro, comprensible y secuencial de la planificación y aplicación del manejo de las cuencas hidrográficas brinda oportunidades a los responsables del manejo de humedales de formular sus contribuciones adecuadamente y relacionarse con la sociedad civil, los usuarios de la tierra y el agua, los responsables de la planificación y el manejo de los recursos hídricos y sus homólogos de los sectores del uso de la tierra. La secuencia exacta es quizás menos importante que el hecho de que exista un proceso establecido que sea oficial, organizado y transparente, en el que puedan participar todos los sectores y grupos pertinentes. Las orientaciones de la Convención relativas a la integración de los humedales en el manejo de las cuencas hidrográficas se exponen en el marco de ese proceso secuencial, el enfoque denominado "camino crítico", que se describe detenidamente en las presentes Orientaciones Consolidadas (Secretaría de la Convención de Ramsar, 2010: 19)

La cita anterior aplica en el estudio debido a que la cuenca del Río Frío posee sistemas de humedales de gran importancia. En la cuenca se encuentran los humedales de Caño Ciego, Caño Blanco y el Refugio de Vida Silvestre Mixto humedal de Cańo Negro. Los efectos en la parte alta de la cuenca afectan directamente estos humedales y han generado impactos ambientales negativos debido a la falta de planificación en las actividades antropogénicas que se desarrollan en el sitio. Se produce entonces una situación contradictoria en la que, a pesar de que la población y los representantes de la sociedad civil desean aumentar la actividad turística en la zona, no se han realizado proyectos para mejorar la gestión de la cuenca; lo anterior conlleva a que cada día disminuya el potencial turístico debido a los impactos asociados con una gestión inadecuada.

\section{Complementariamente, GWP cita que:}

... los gobiernos nacionales establecen las políticas para el uso y protección de los recursos hídricos en un país. Si bien la implementación de dichas políticas es eficaz en muchas escalas, allí donde se implementan políticas a escala de cuenca, existe la oportunidad de generar soluciones para "toda la cuenca" y resolver controversias aguas arriba, aguas abajo (para un río) y de región a región (para un lago o el agua subterránea). El enfoque de "toda la cuenca" permite la evaluación de un impacto a nivel de sistema. En otras palabras, las políticas nacionales, así como también los acuerdos internacionales y los convenios regionales para aguas transfronterizas, se aplican en cuencas naturales. La relación que existe entre la gestión de los recursos hídricos dentro de un país y la gestión del agua en cuencas se vuelve, de esta manera, dinámica y más sensible a las circunstancias cambiantes, sean estas ambientales, sociales o económicas (GWP, 2009: 10). 
La cita anterior introduce un concepto clave para la discusión que se centra en evaluar las cuencas o zonas de estudio desde el enfoque de los sistemas naturales. A continuación se presenta información sobre este concepto y su relevancia.

\subsection{El uso de sistemas para la comprensión del manejo de cuencas y turismo}

La teoría de sistemas es útil en muchos tipos de investigación y en muchos campos de estudio debido a que su uso provee al investigador de distintos enfoques para comprender las realidades a las que se enfrenta. El término sistema turístico se refiere a los elementos que se han extraído de la actividad turística como centrales y que se han llevado a conceptos.

Arnold y Osorio apuntan que:

... la primera formulación en tal sentido es atribuible al biólogo Ludwig von Bertalanffy (1901-1972), quien acuñó la denominación "Teoría General de Sistemas". Para él, la TGS debería constituirse en un mecanismo de integración entre las ciencias naturales y sociales y ser al mismo tiempo un instrumento básico para la formación y preparación de científicos (Arnold, y Osorio, 1998: 41).

Lohmann y Panosso señalan que:

... la teoría de los sistemas es nueva y que tiene sus inicios en la década de 1930 . El propósito del uso de la teoría de sistemas es permitir comprender situaciones que son complejas y están conformadas por muchas variables que se afectan entre sí y afectan las interacciones con el medio. Por medio de la desagregación en sistemas es posible reducir la complejidad de un problema y facilitar la comprensión y búsqueda de soluciones (Lohmann y Panosso, 2016: 1).

Por otra parte, Jiménez (2009: 3) expresa que "de acuerdo con la utilización del andamiaje conceptual de la Teoría General de Sistemas (TGS), cualquier abstracción de la realidad (corte de la realidad) pudiera ser un sistema, siempre y cuando se definan claramente los parámetros dentro de los cuales se establece tal sistema”. Se debe señalar aquí la diferencia entre pensamiento sistémico y la teoría general de sistemas. En el primer caso se considera una manera de pensar que busca todos los elementos constituyentes (pensamiento integral u holístico); en el segundo, se requieren definiciones más precisas para poder operar con vínculos claros y útiles, así como para impedir que su identidad se diluya dentro de un conjunto difuso de teorías, métodos y técnicas.

Lo anterior obliga a la definición de conceptos y a describir la forma en la que aplican, de manera específica, los componentes del hecho estudiado y definido como tal sistema, así como la forma en la que opera. La 
tabla 1 resume algunas definiciones sobre sistema turístico que se encuentran en la literatura:

Tabla 1

Resumen de algunas definiciones de teoría de sistemas turísticos

\begin{tabular}{|l|l|}
\hline \multicolumn{1}{|c|}{ Fuente } & \multicolumn{1}{c|}{ Definición } \\
\hline OMT, 1993 & $\begin{array}{l}\text { Actividad multisectorial que comprende: actividades y puntos de } \\
\text { interés, ya sean naturales o artificiales; servicios e instalaciones de } \\
\text { alojamiento, otros servicios que no son de alojamiento como ali- } \\
\text { mentación, artesanías, cambio de moneda, bancos, servicios médi- } \\
\text { cos, seguridad, comunicaciones; transporte; infraestructura de } \\
\text { servicios básicos; elementos institucionales y políticas públicas. }\end{array}$ \\
\hline Aranzazu, 2003 & $\begin{array}{l}\text { La autora señala que, según la OMT, la actividad turística es el } \\
\text { resultado complejo de interrelaciones entre diversos factores que } \\
\text { hay que considerar conjuntamente desde una óptica sistémica, es } \\
\text { decir, un conjunto de elementos operadores del mercado interrela- } \\
\text { cionados entre sí que evolucionan dinámicamente. El sistema tu- } \\
\text { rístico está formado por la oferta, la demanda, el espacio geográfico } \\
\text { y los operadores de mercado. }\end{array}$ \\
\hline Leiper, 1979 & $\begin{array}{l}\text { El sistema turístico es el marco de referencia del turismo. Signifi- } \\
\text { cando este último el movimiento temporal de personas desde su } \\
\text { lugar de residencia habitual a otros sitios por más de una noche. Se } \\
\text { consideran los elementos del turismo las regiones generadoras, las } \\
\text { rutas de tránsito, la región de destino y la actividad turística en sí. }\end{array}$ \\
\hline
\end{tabular}

Fuente: elaborado por los autores.

En resumen, el análisis sistémico permite tener una visión general de las interacciones de los componentes del territorio. El ser humano y sus interacciones deben considerarse como un subsistema de gran potencial para impactar; lo anterior conduce al tema de la participación de las comunidades en los procesos de gestión de cuencas.

\subsection{La participación de base local en el manejo de cuencas}

El tema de participación comunal en los procesos de gestión de cuencas ha sido investigado por autores como Guerrero et al. (2010), CATIE (2009), Gaspari et al. (2013), Lozano et al. (2008) y Comcure (2010).

La falta de coordinación interinstitucional y de trabajo con las comunidades ha sido identificada como un factor que influye negativamente en las iniciativas de gestión de cuencas, por ejemplo, Guerrero et al. (2010) realizaron un análisis de uso del recurso hídrico en una cuenca en México; encontraron que la falta de coordinación entre los diferentes niveles de gobierno, la ausencia de integración entre instituciones y la participa- 
ción ciudadana de actores locales rurales tienen como consecuencia que no exista un manejo adecuado de los recursos hídricos. Por tanto, es importante fomentar las organizaciones rurales, crear fortalezas locales internas, así como concientizar sobre la problemática ambiental, ello permitirá que los grupos rurales generen acciones y políticas de desarrollo y conservación. En la figura 1 se resumen las líneas de trabajo que deben realizarse en el tema de gestión de cuencas para fortalecer el turismo sostenible desde la base comunal.

\section{Figura 1 \\ Resumen de las líneas de trabajo de la gestión de cuencas para fortalecer el turismo sostenible de base comunal}

Líneas de trabajo de la gestión de cuencas para fortalecer el turismo sostenible de base comunal

Uso de enfoque sistémico para el análisis de la cuenca:

En el análisis de diagnóstico se deben integrar todos los elementos (bióticos y abióticos) de la cuenca, además, establecer las interacciones entre los elementos internos y externos del sistema. El turismo debe considerarse un subsistema, cuya interacción dentro de la cuenca puede influenciar y causar impactos a otros subsistemas.

\section{Ordenamiento territorial y proyectos complementarios:}

Las herramientas de ordenamiento territorial de cada nación permiten diferentes grados de impacto en los territorios, dependiendo de la legislación éstas se deben aplicar y complementarse con proyectos que solucionen problemas cuyo ámbito de acción se encuentra fuera de las potestades de la legislación.

Determinar los servicios ecosistémicos que ofrece la cuenca:

Dependiendo de la situación específica de cada cuenca se debe valorar el aporte de la zona como generadora de servicios ecosistémicos, fuente de recursos hídricos para varios usos, recurso paisajístico, herramienta de fijación de carbono en plantas y suelo, control de erosión, corredor biológico, hábitat de especies objeto de conservación nacionales e internacionales, zonas con atractivos turísticos culturales o naturales entre otros.

Proceso participativo de los actores locales como base:

Las personas que viven en la cuenca serán los principales beneficiados de su gestión, por lo que es imprescindible que logren involucrarse en las iniciativas que se desarrollen; se debe realizar un mapeo de actores y generar un programa de participación comunal para crear proyectos de base comunal que tengan el mayor impacto positivo en la cuenca .

Fuente: elaborado por los autores. 


\section{Metodología}

La investigación se generó utilizando las siguientes herramientas:

- Análisis de los atractivos turísticos existentes en el cantón de Guatuso y su zona vecina; la metodología utilizada fue la establecida por el Centro Interamericano de Capacitación Turística (Cicatur, 1979); agrupa los atractivos en cinco categorías básicas: sitios naturales, museos y manifestaciones culturales, actividades tradicionales, realizaciones técnicas, científicas o artísticas contemporáneas y acontecimientos programados.

- Se determinó el estado de la demanda y oferta en el sistema turístico.

- Por medio de trabajo de campo y fichas de levantamiento de información se estableció el estado de la infraestructura y superestructura turística

- Con información de mapas del Ministerio de Agricultura y Ganadería de Costa Rica (MAG) (escala 1:50,000, año 2000; escala 1:200,000, año 1990) y la Fundación Neotrópica (escala 1:50,000, año 1994) se determinó la capacidad de uso del suelo del cantón.

- Se generó el Modelo de Elevación Digital (MED) del cantón. Para este proyecto se recurrió a un método de interpolación. Como base de datos inicial se utilizaron Curvas de nivel; escala 1:50,000 provenientes de los mapas oficiales generados entre los años sesenta y setenta por el Instituto Geográfico Nacional (IGN). La elevación que reporta cada curva de nivel se interpola mediante una herramienta contenida en el software ArcGIS, llamada "de topo a ráster"; la misma está basada en el programa ANUDEM ${ }^{1}$. El resultado de este proceso es un archivo en formato ráster, equivalente a una hoja cuadriculada donde cada cuadro (píxel) tiene un valor asignado que corresponde a la elevación promedio en el área que abarca el cuadro. La dimensión del ráster y de los píxeles que lo conforman se define en el proceso de creación del mismo, para este proyecto se usó una extensión (dimensión) superior al rectángulo que contiene al distrito, para tener mayor información contextual, y un tamaño de píxel de cinco metros de lado. Esta base de datos es

\footnotetext{
${ }^{1}$ La herramienta "de topo a ráster" es un método de interpolación diseñado específicamente para crear modelos digitales de elevación (DEM) hidrológicamente correctos. La versión actual de ANUDEM utilizada en ArcGIS es 5.3. Esta herramienta interpola los valores de elevación para un ráster mientras se imponen restricciones que aseguran: 1) Una estructura de drenaje conectado; 2) La correcta representación de crestas y arroyos a partir de los datos de curvas de nivel de entrada. Como tal, es el único interpolador de ArcGIS diseñado específicamente para trabajar de forma eficaz y eficiente con información lineal como curvas de nivel.
} 
conocida como MED (Modelo de Elevación Digital) o MDT (Modelo Digital de Terreno).

- El mapa de uso del suelo se confecciona a partir de un análisis fotogramétrico, el cual consiste en la corrección, deformación, ubicación y comprensión de fotografías aéreas o imágenes derivadas de sensores remotos; para este estudio se tomaron en consideración dos fuentes de información: imágenes satelitales LANDSAT 8, capturadas durante el 2014. Escala aproximada 1:55,000. Basemap, disponible en el paquete ArcGIS; resolución de píxel hasta menos de un metro.

- Posteriormente se identificaron las cuencas presentes en el cantón y se determinó el uso del suelo en la cuenca.

- Se realizaron giras de campo para corroborar los usos del suelo en el cantón y recorridos en el cauce del Río Frío para comprobar los problemas de navegabilidad. El proceso también incluyó la realización de talleres con actores clave y entrevistas.

\section{Resultados}

\subsection{Sistema turístico}

El análisis del sistema turístico del cantón incluyó el desarrollo del perfil del productor turístico, a continuación se presentan los resultados:

- Producto turístico para no residentes: paquete de un día de visita a Río Celeste/Parque Nacional Volcán Tenorio (PNVT). Este producto comprende el hospedaje en hoteles en San José, Alajuela, Heredia, La Fortuna y Guanacaste. Estos recorridos de un día incluyen el transporte y la alimentación. Los visitantes son buscados en sus hoteles, transportados hasta el PNVT, donde permanecen alrededor de cuatro horas, posteriormente se procede a almorzar y se regresa a la zona de hospedajes.

- Producto turístico para residentes: visita a Río Celeste/PNVT. En un viaje de ida y vuelta se visita el PN. Está diseñado para salir desde San José, pasando por sectores de Heredia y Alajuela y desde Fortuna.

- Turismo de aventura: tubbing en el Río Celeste, desarrollado por varias empresas de la zona y del valle central. Diseñado para que los visitantes disfruten el viajar en un neumático inflado por las aguas del Río Celeste. Hay facilidad para incluir el servicio de alimentación. La información se obtuvo en trabajo de campo y 
búsquedas en internet basadas en paquetes turísticos que se ofrecen en el cantón.

- Turismo para nacionales en los márgenes del Río Celeste: existen proyectos enfocados al turismo nacional; el atractivo es disfrutar de un baño y días de campo, así como acampar en los márgenes de Río Celeste.

- Turismo cultural en el territorio indígena Maleku: algunos de los proyectos se enfocan en recibir visitantes no residentes, otros se están enfocando en recibir grupos de estudiantes de universidades costarricenses.

- Hoteles con paquetes turísticos todo incluido en las cercanías de PNVT: se ofrecen paquetes de tres días y dos noches con énfasis en la visita al PNVT y al spa, en un paquete de naturaleza y bienestar, así como paquetes de cuatro días y tres noches diseñados para luna de miel con énfasis en ecoturismo, se visita PNVT y Caño Negro.

\subsection{Atractivos turísticos}

Según UNA (2012) y ProDUS-UCR (2015), los atractivos turísticos identificados en el cantón fueron: Parque Nacional Volcán Tenorio, proyectos de turismo cultural ubicados en el territorio indígena Maleku, humedales de Caño Ciego, Árbol de la paz, Río Frío, Río Celeste, paisaje rural, refugio nacional de vida silvestre Caño Negro, agroturismo, turismo rural, bosques de marillales cedro María (Calophyllum brasilense) y palma real (Attalea butyracea), yolillanes (palma yolillo Raphia taedige$r a)$, patrimonio intangible, producción e historia del cacao en el cantón, lago Cote.

\subsection{Infraestructura turística}

Se encontraron 18 sitios de hospedaje y 31 de venta de alimentos, sin embargo, la mayoría de estos establecimientos están destinados a la atención de agentes de ventas que pasan por la zona, hay muy pocos enfocados en el turismo. El precio promedio por habitación doble fue de \$30 USD.

\subsection{Topografía y pendientes}

Las siete categorías de relieve que se utilizaron son: relieves planos para los terrenos con pendientes entre $0-2 \%$, los relieves ligeramente ondulados para pendientes con valores entre $2-5 \%$, terrenos ondulados para rangos de pendientes entre $5-15 \%$, relieves fuertemente ondulados son 
aquellos con pendientes entre 15-30\%, relieves escarpados poseen pendientes entre $30-50 \%$, relieves fuertemente escarpados son los terrenos con valores de pendientes entre 50-75\%, y los relieves montañosos son aquellos con pendientes mayores a 75\%. En la gráfica 1 se muestra la distribución de pendiente para cada distrito.

Las pendientes del cantón son mayores en las zonas cercanas al Parque Nacional Volcán Tenorio, ubicado al sureste del cantón, la topografía de la zona va disminuyendo hasta el noreste del cantón, donde se llega al Refugio Nacional de Vida Silvestre Caño Negro. La zona intermedia

\section{Gráfica 1}

Representación topográfica; distribución del área que ocupan las pendientes agrupadas por distrito, cantón Guatuso

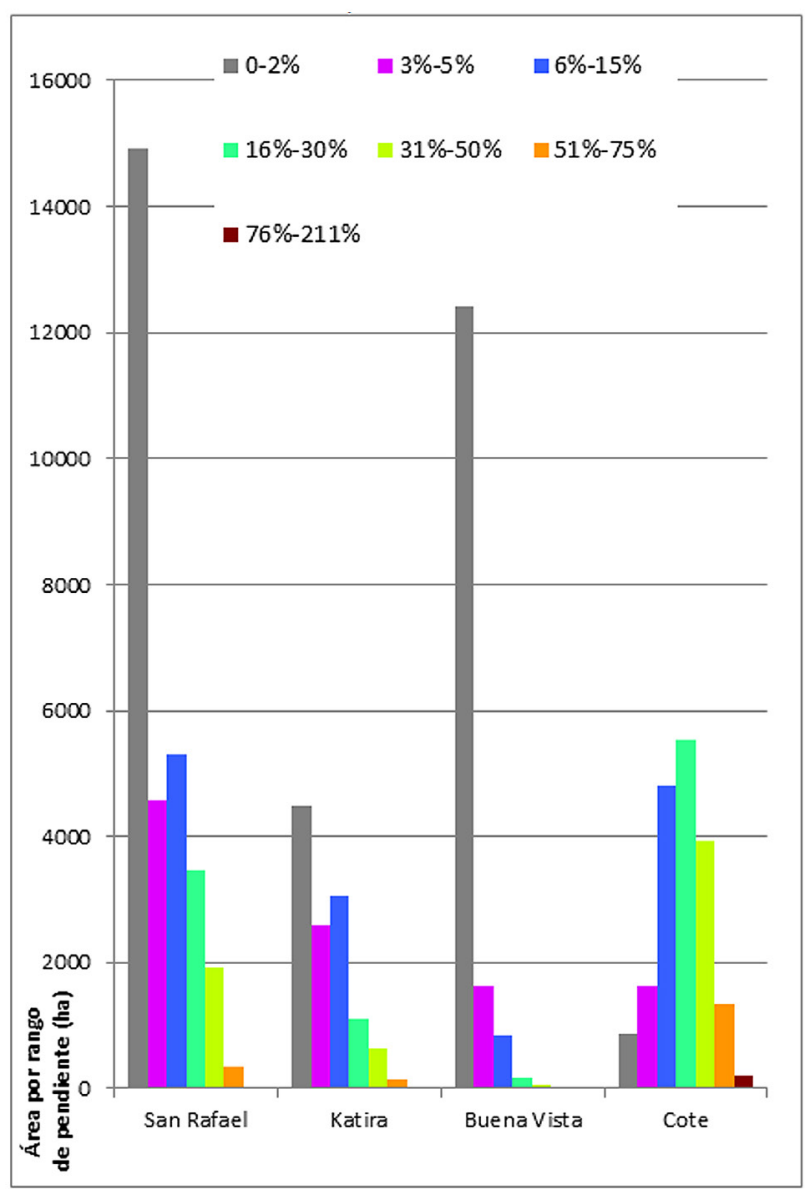

Fuente: ProDUS-UCR (2015). 
entre las dos áreas protegidas posee en su mayoría uso del suelo dedicado a los pastizales para ganadería bovina.

\subsection{Cobertura boscosa y uso del suelo}

En la tabla 2 se presenta el resultado del análisis de uso del suelo para el cantón de Guatuso.

Debido a la escala de la fotografía disponible con certeza de fecha (Landsat) hay una incertidumbre considerable en los porcentajes de pastos y cultivos, pues ambos muestran texturas similares en la foto, por lo que es posible que algunos pastos ganaderos se confundan con cultivos herbáceos y viceversa; por ello se decidió conjugarlos en la misma categoría. Sin embargo, como medida de corroboración se compararon los datos de producción agropecuaria y los reportados por la propuesta de Plan Regulador del ańo 2012. Con estos datos se estima la existencia de 3200 hectáreas de cultivos, correspondientes con un 4.2 de la superficie del cantón, lo que representa, para pastos y arboles dispersos, 46.8 del total del cantón.

La presencia de bosque en el cantón de Guatuso se concentra principalmente en los sectores del Parque Nacional Volcán Tenorio y el Refugio de

Tabla 2

Uso del suelo en la zona de estudio, según fotografía 2014 (por distritos)

\begin{tabular}{|l|r|r|r|r|r|r|}
\hline $\begin{array}{l}\text { Capacidad agricola- } \\
\text { forestal }\end{array}$ & $\begin{array}{c}\text { San } \\
\text { Rafael }\end{array}$ & Katira & \multicolumn{1}{c|}{$\begin{array}{c}\text { Buena } \\
\text { Vista }\end{array}$} & \multicolumn{1}{c|}{$\begin{array}{c}\text { Cote } \\
\text { (hectáreas) }\end{array}$} & $\begin{array}{c}\text { Área } \\
\text { cantón }\end{array}$ & \multicolumn{1}{c|}{ (\%) } \\
\hline Cobertura forestal & 8.541 & 4.241 & 2.389 & 13.526 & 28.693 & 38 \\
\hline $\begin{array}{l}\text { Pastos y árboles } \\
\text { dispersos-cultivos }\end{array}$ & 20.307 & 7.440 & 6.256 & 4.647 & 38.650 & 51 \\
\hline Urbano & 334 & 200 & 48 & 44 & 627 & 1 \\
\hline Desnudo & 28 & - & - & - & 28 & 0,0 \\
\hline Cuerpos de agua & 258 & 34 & 44 & 1,5 & 337 & 0,4 \\
\hline Nubes y sombras & 714 & 8 & 6.303 & - & 7.025 & 9 \\
\hline Humedales & 237,1 & 34 & 0,3 & 91 & 362 & 0,5 \\
\hline Total general & 30.419 & 11.959 & 15.039 & 18.305 & 75.722 & 100 \\
\hline
\end{tabular}

Fuente: ProDUS-UCR (2015). 


\section{Mapa 1 \\ Uso de uso del suelo del cantón de Guatuso}

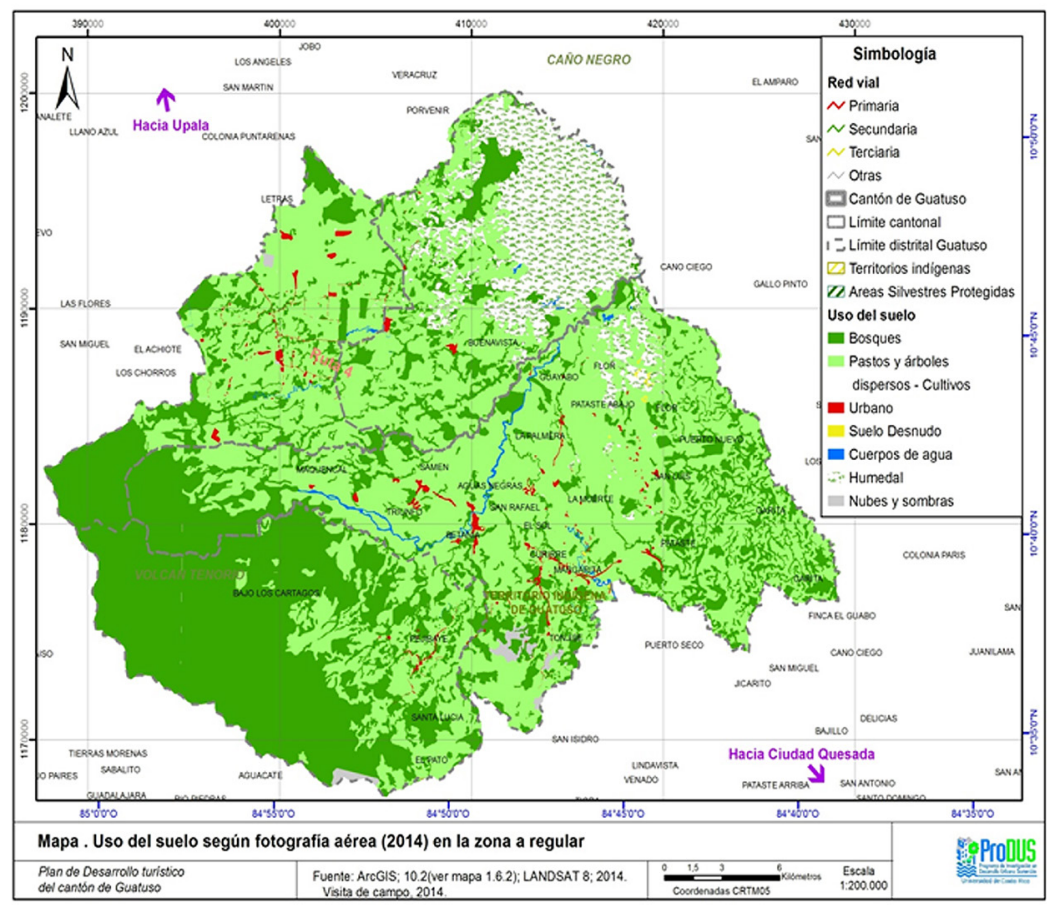

Fuente: ProDUS-UCR, 2015.

Vida Silvestre Caño Negro como se observa en el mapa 1. A lo largo del cantón se da una cobertura boscosa dispersa, principalmente rodeada de pastos o extensiones de tierra dedicadas a la agricultura.

En la tabla 3 se resume la información de cobertura boscosa, pastos, pastos con árboles y cultivos presentes en Guatuso. La información de la tabla 3 es coherente con la imagen que se presenta en el mapa 1 , donde el paisaje predominante son los pastos con árboles dispersos. Los pastos para la actividad ganadera extensiva cubren la zona que se ubica entre del Parque Nacional Volcán Tenorio (parte de mayor elevación de la cuenca) y la zona baja de la cuenca cubierta por humedales.

En las giras de reconocimiento se verificó la deforestación que se ha dado en el cantón, donde se desarrolla principalmente la actividad ganadera que ha desplazado la existencia de bosque primario para la explotación de la tierra. En la figura 2 se observa un ejemplo del paisaje que se puede encontrar en el cantón de Guatuso, producto de la deforestación. La existencia de zonas boscosas representa un potencial turístico como atrac- 
Tabla 3

Cobertura boscosa en el cantón de Guatuso

\begin{tabular}{lrr}
\hline \multicolumn{1}{c}{ Categoria } & Área $\left(\mathrm{km}^{2}\right)$ & $\begin{array}{c}\text { Porcentaje de } \\
\text { cobertura }\end{array}$ \\
\hline Forestal & 176.84 & $23.28 \%$ \\
Humedal & 45.12 & $5.94 \%$ \\
Cuerpo de agua & 5.26 & $0.69 \%$ \\
Pasto & 120.23 & $15.82 \%$ \\
Pasto con árboles & 376.91 & $49.61 \%$ \\
Cultivos & 32.63 & $4.29 \%$ \\
Urbano & 2.23 & $0.29 \%$ \\
Total & 759.76 & $100.00 \%$ \\
\hline
\end{tabular}

Fuente: UNA, (2012).

tivo para el disfrute del paisaje, por lo que se debe pensar en desarrollar programas de reforestación del cantón para la recuperación del bosque.

La deforestación también se ha dado en las zonas de protección de los ríos ocasionando erosión en sus márgenes. En la figura 3 se observa la falta de árboles en las orillas del río como soporte del suelo. En el caso del Río Frío el desprendimiento de material dificulta su navegación. La

Figura 2

Zonas boscosas dentro del cantón de Guatuso

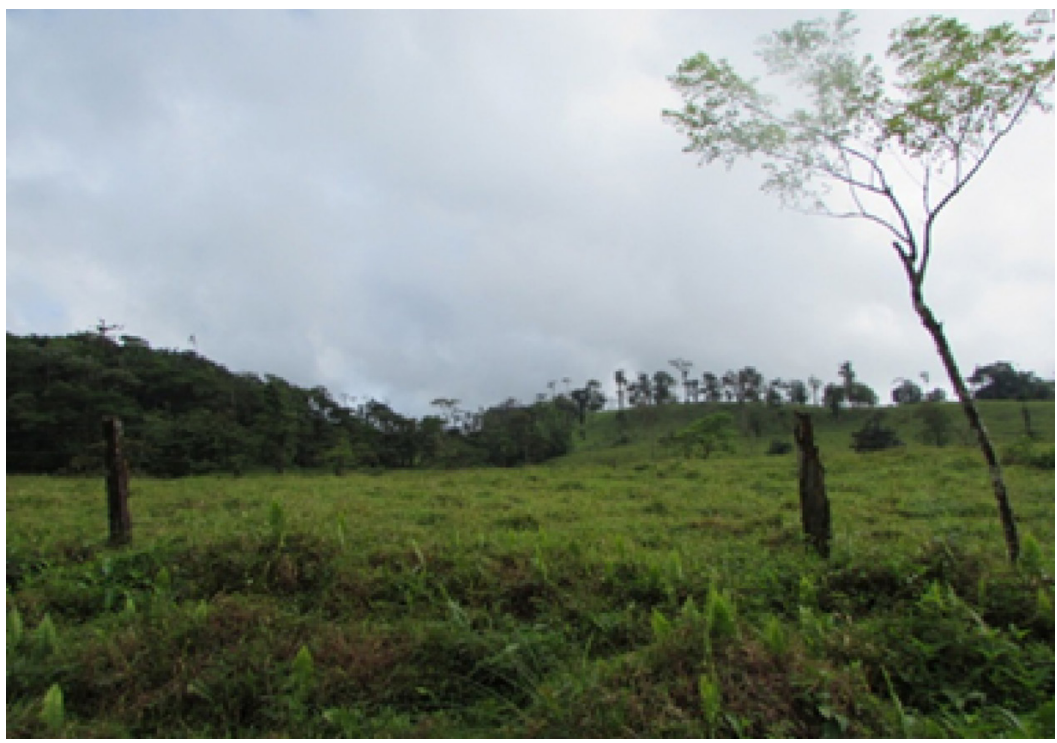

Fuente: ProDUS-UCR (2015). 


\section{Figura 3}

\section{Deforestación en los márgenes del Río Frío}
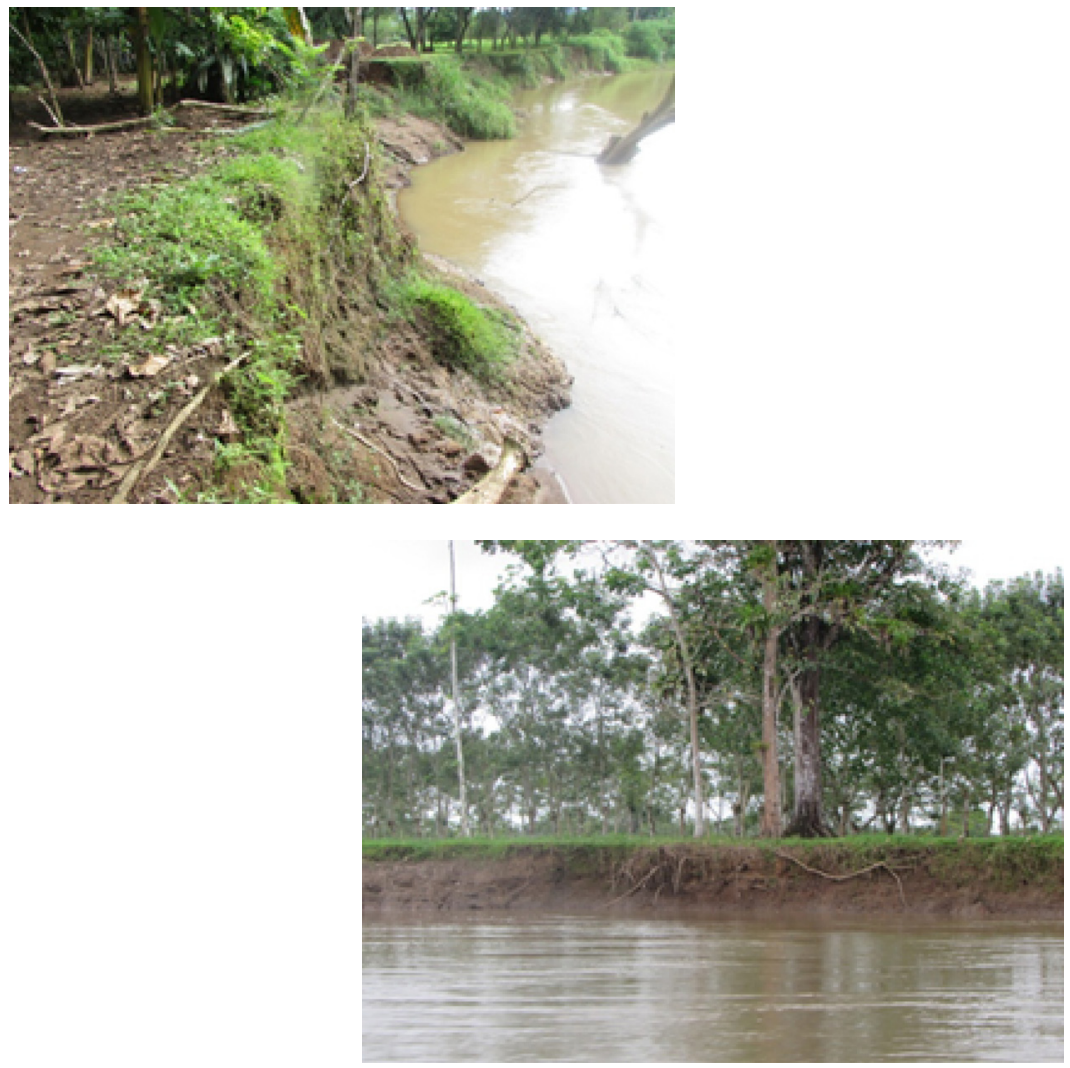

Fuente: ProDUS-UCR (2015).

presencia de materiales en los ríos afecta la calidad del agua y puede generar inundaciones debido a la obstrucción del paso del agua.

La falta de permeabilización en los suelos contribuye a que se arrastren sedimentos a los cauces de los ríos, esto ocurre muchas veces debido a la deforestación o al sobreuso del suelo. En Guatuso este problema se encuentra asociado al desarrollo intensivo de la actividad agropecuaria y a las técnicas inapropiadas de manejo, lo que contribuye a que se pierda la capa fértil de la tierra, que eventualmente desencadena la erosión y un posible arrastre de sedimentos a los ríos.

En el caso de Guatuso, el río que se ve principalmente afectado por el problema de la sedimentación es el Río Frío, en la figura 4 se observa el material que se encontró en el cauce del río en una de las giras de reconocimiento, además de las raíces expuestas de muchos árboles en los márgenes del río, muestra de la erosión que se ha dado en el lugar. 
La caída de árboles al río se produce por la pérdida de soporte al lavarse las raíces, principalmente cuando el río crece o hay precipitaciones. $\mathrm{Al}$ no existir vegetación que lo proteja el suelo termina desprendiéndose y depositándose en el río. El problema de la sedimentación se origina en la parte alta del cantón cerca del Volcán Tenorio, donde debido a la deforestación se favorece al arrastre de materiales hasta el río.

Adicionalmente, dicha sedimentación produce un efecto directo sobre la desembocadura del Río Frío en el humedal Caño Negro, unido al efecto de los agroquímicos vertidos en el río, producto de la actividad agrícola. Esto implica una seria afectación sobre este ecosistema.

\section{Figura 4 \\ Sedimentación en el Río Frío}
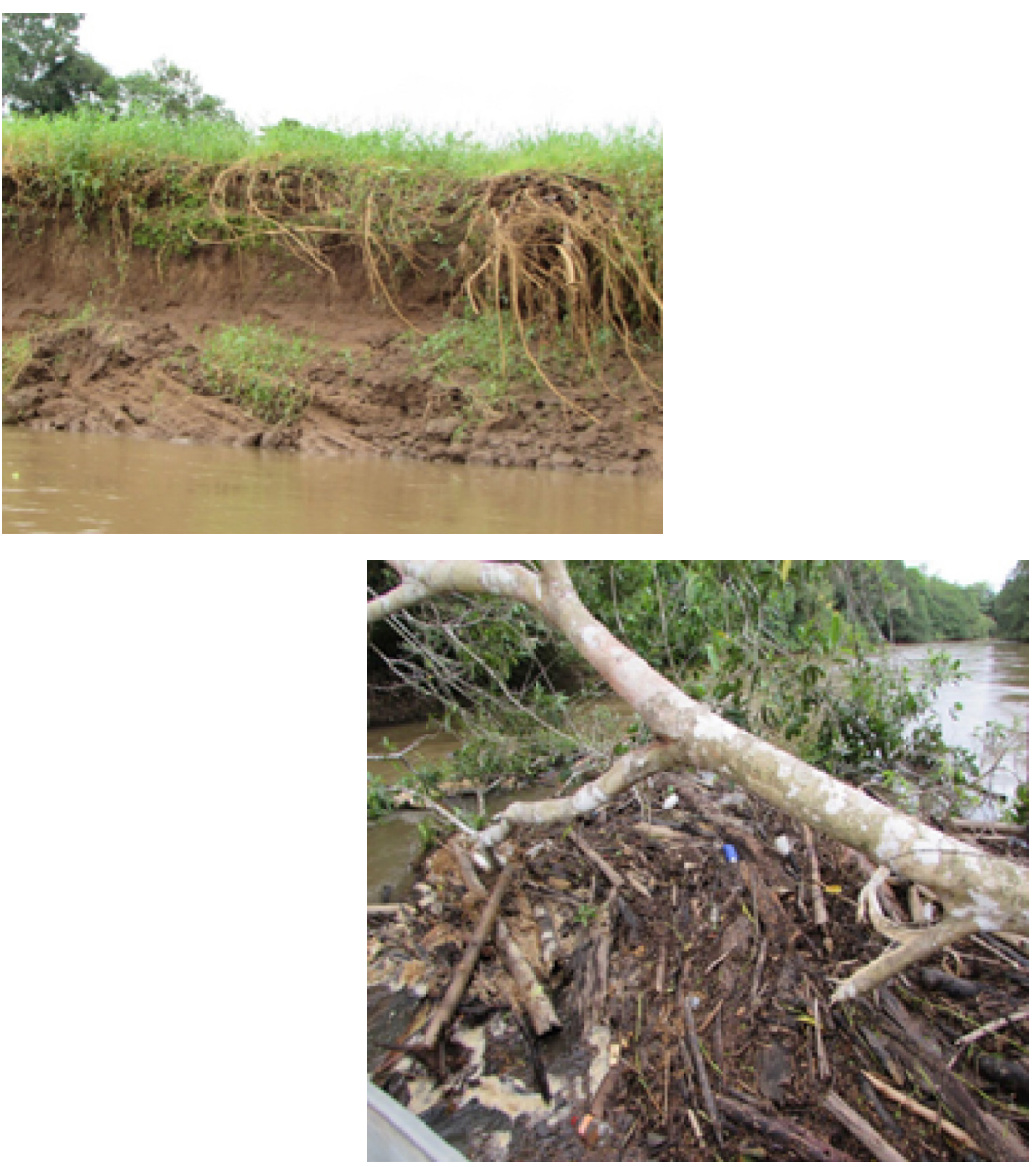

Fuente: ProDUS-UCR (2015). 
El problema de la sedimentación y erosión de márgenes en el Río Frío ha generado que este cauce pierda su capacidad para la navegabilidad, por lo que no se pueden realizar viajes en lancha desde San Rafael de Guatuso hasta el Refugio de Vida Silvestre de Caño Negro.

\subsection{Cuenca del Río Frío, uso y estado}

La cuenca del Río Frío se puede dividir en cuatro secciones:

Sector suroeste-Parque Nacional Volcán Tenorio: se le señala en el mapa como microcuenca del Río Frío, la cual tiene su punto de control en el puente ubicado en el centro de San Rafael. Este sector es de gran importancia debido a que desde él se puede ejercer un mayor control sobre las zonas de recarga de los acuíferos. Se deben ejercer controles sobre el área de transición del parque, ya que es el sitio donde se ubica la mayoría de fuentes de abastecimiento de agua potable del cantón de Guatuso.

Sector sureste: se ubica fuera del cantón y fuera del área protegida del Parque Nacional Volcán Tenorio, por lo que no se puede establecer un control en cuanto a uso de suelo y cobertura boscosa sobre las partes altas de la cuenca.

Sector centro-norte: este sector corresponde a la parte más deforestada de la cuenca, en la cual se desarrollan actividades agropecuarias, las cuales tienen gran influencia sobre la parte baja en cuanto a arrastre de sedimentos y a afectación por el uso de agroquímicos.

Sector norte-humedal de Caño Negro: es la parte más baja de la cuenca y se ve conformada por el humedal de Caño Negro, el humedal de Caño Ciego y el humedal de Caño Blanco. Es el área más vulnerable, ya que depende del manejo que se le haya dado a la cuenca aguas arriba.

En el tema de ordenamiento territorial, el cantón de Guatuso no cuenta con Plan Regulador, en el ańo 2012 la Universidad Nacional de Costa Rica elaboró una propuesta pero no se logró oficializar e implementar el proyecto. Esta es una de las principales debilidades de gestión territorial de la zona que se considera afecta la competitividad del cantón. Sobre este tema Zumbado (2011) expone la importancia de contar con planes reguladores para lograr que las zonas rurales mejoren sus condiciones de producción socioeconómica, sin que esto signifique un detrimento en los indicadores ambientales de la zona. Es importante generar una línea base de las condiciones ambientales y de ella partir para de- 
terminar que actividades, según su tamaño y condiciones específicas, pueden mejorar o ir en detrimento de esta condición. En el caso del cantón de Guatuso, generar un manejo de cuencas mejorará los indicadores ambientales y también ayudará a fortalecer el sistema turístico, de ahí la importancia que tiene para los gobiernos locales considerar relación de ordenamiento territorial y turístico en sus territorios.

\subsection{Afectación al sistema turístico}

La navegabilidad del Río Frío se ha perdido en la mayor parte de los tramos, los botes tienen problemas debido a que se quedan atascados en el fondo; debido a la caída de árboles es necesario llevar equipo pesado como motosierras para lograr cortar los troncos y realizar los viajes.

Los vecinos de la zona reportan que de los años cincuenta a los setenta se realizaban viajes con botes de gran tamaño por el río, se transportaban personas y mercaderías hasta Nicaragua. Debido al problema mencionado no es posible que los turistas puedan embarcar en San Rafael de Guatuso y de ahí realizar recorridos a Caño Negro, aunque esto generaría una diversificación económica de importancia en la zona.

Con la habilitación de la navegación se podrían ofrecer recorridos de pesca artesanal, observación de flora y fauna, recorridos hasta Caño Negro y recorridos culturales por el río para comprender ese elemento dentro de la cosmovisión de la cultura indígena Maleku.

La falta de gestión en la zona ha generado graves impactos ambientales, además de afectar la navegabilidad en el Río Frío; se reporta que el arrastre de sedimentos también está afectando los humedales de Caño Negro. Los sedimentos han generado que las lagunas pierdan profundidad y se dificulte su navegación. Otro de los impactos que se generan es la afectación de la biodiversidad acuática de los hábitats presentes en el sitio.

Todos los puntos anteriores demuestran cómo el enfoque de sistemas es necesario para comprender las interacciones entre los sistemas naturales y los sistemas antropogénicos, como el turístico.

\section{Conclusiones}

Turismo, gestión de cuencas y su impacto:

- La gestión de cuencas como herramienta de fortalecimiento del turismo de base comunal fortalecerá el sistema turístico de una zona rural mediante el mejoramiento paisajístico del sitio, incremento de biodiversidad, control de erosión y deforestación, fo- 
mento a la conectividad entre áreas silvestres protegidas, apoyo a la gestión de los corredores biológicos, fortalecimiento de las zonas de amortiguamiento de áreas protegidas, apoyo a la lucha contra el cambio climático, incremento de oportunidades de encadenamientos entre el sector agropecuario y turístico, y gestión integral del recurso hídrico para su uso en consumo humano, navegación, actividades acuáticas y transporte.

- En el caso en estudio, la deforestación para la apertura de espacios de cultivo o ganadería es el mayor causante del sobreuso del suelo en el cantón de Guatuso. La pérdida de la cobertura forestal es una acción que desencadena una serie de impactos negativos en el corto, mediano y largo plazo. Dentro de estos impactos se incluye la pérdida de biodiversidad, aumento de la posibilidad de contaminación de cauces de agua, alteración de las unidades de paisaje natural, pérdida de fuentes de fijación de $\mathrm{CO}_{2}$, posible afectación de zonas de recarga de acuíferos y, por consiguiente, disminución del agua disponible para actividades humanas como el turismo.

- El cambio de uso del suelo ha generado que se ubiquen actividades agropecuarias en espacios que deben dedicarse únicamente a la protección de los recursos naturales. El ser humano debe lograr un equilibrio entre la producción agropecuaria y la preservación de los recursos naturales.

- Los ríos del cantón de Guatuso presentan sedimentación relacionada con la erosión por el sobreuso del suelo. Este aspecto está afectando la navegabilidad de los cauces y por ende la posibilidad de efectuar turismo en la zona.

- En el caso de Costa Rica, los gobiernos locales, en uso de sus facultades como administradores de los intereses locales, pueden generar proyectos, reglamentos especiales y normativa urbanística tendiente al resguardo y recuperación de los recursos forestales, en coordinación con las competencias dadas a otros órganos del Estado.

- Se debe implementar urgentemente un Plan de Manejo Integral de la cuenca del Río Frío, en Costa Rica existen experiencias exitosas en el tema, es el caso de la Comisión para el Ordenamiento y Manejo de la Cuenca del Río Reventazón (Comcure); después de catorce ańos, Comcure ha acumulado una serie de logros entre los que destacan: 2500 fincas involucradas en programas de conservación de suelos, 350 biodigestores instalados, 7 estaciones hidrometeorológicas y una fluviográfica, aumento de $11 \%$ de la cobertura vegetal del 2000 al 2009, lo cual equivale a 16,000 hectáreas de nueva vegetación. Este ejemplo de gestión exitosa de cuenca gene- 
ra un punto de partida que debería estudiarse para lograr identificar las acciones inmediatas a desarrollar en la cuenca del Río Frío en Guatuso.

\section{Fuentes consultadas}

Aranzazu, Rita (2003), Formación para líderes de turismo: herramientas para la gestión sostenible de turismo, Servicio Nacional de Aprendizaje, Bogotá, Colombia.

Arnold, Marcelo y Francisco Osorio (1998), "Introducción a los conceptos básicos de la teoría general de sistemas", Revista Cinta moebio, núm. 3, Facultad de Ciencias Sociales Universidad de Chile, Santiago Chile, Chile, pp. 41.

CATIE (Centro Agronómico Tropical de Investigación y Enseñanza) (2009), El enfoque de género en la gestión y manejo de cuencas hidrográficas. El caso de la subcuenca Aguas Calientes, Nicaragua, CATIE, Turrialba, Costa Rica.

Cicatur (Centro Interamericano de Capacitación Turística) (1979) Metodología de inventarios turísticos, Cicatur-OEA, Ciudad de México, México.

Comcure (Comisión para el Ordenamiento y Manejo de la Cuenca del Río Reventazón) (2010), La importancia del manejo de cuencas, Comcure, San José, Costa Rica.

Gaspari, Fernanda, Alfonso Rodríguez, Gabriela Senisterra, María Delgado y Sebastián Besteiro (2013), Elementos metodológicos para el manejo de cuencas hidrográficas, Universidad Nacional de la Plata, Buenos Aires, Argentina.

GWP (Global Water Partnership) (2009), Manual para la gestión integrada del recurso hídrico en cuencas, GWP Editorial, Estocolmo, Suecia.

Guerrero, Aída, Peter Gerritsen, Luis Martínez, Silvia Salcido, Demetrio Meza, Humberto Bustos (2010), "Governance and social participation in the management of water in el Cangrero micro basin, municipality of Autlén de Navarro, Jalisco, México", Economía, 
Sociedad y Territorio, X (33), El Colegio Mexiquense A. C., Toluca, México, pp. 541-547.

Lohmann, Gul y Alexandre Panosso (2016), Tourism theory: concepts, model and systems, Centre for Agricultural Bioscience International, Londres, Inglaterra.

Jiménez, Alfonso (2009), Una aproximación sistémica al turismo: implicaciones para la multi y la transdisciplinariedad, Universidad del Caribe, Quintana Roo, México.

Leiper, Neil (1979), "The framework of tourism. Toward a definition of Tourism, tourist and the tourist industry", Annals of tourism research, 6 (4), Elsevier, Amsterdam, Holanda, pp. 385-518.

Lozano, Juan, Patricia Mora, Ricardo Lozano, Mauricio Cabrera (2008), Guía técnica cientifica para la ordenación y manejo de cuencas hidrográficas en Colombia. Bogotá, Ministerio de Ambiente y Desarrollo Sostenible, Bogotá, Colombia.

OMT (Organización Mundial del Turismo) (1993), Desarrollo turístico sostenible. Guía para planificadores locales, OTM, Madrid, España.

ProDUS-UCR (Programa de Investigación en Desarrollo Urbano Sostenible-Universidad de Costa Rica) (2015), Plan de Desarrollo Turistico del cantón de Guatuso, ProDUS, Universidad de Costa Rica, San José, Costa Rica.

UNA (Universidad Nacional de Costa Rica) (2012), Plan Regulador del cantón de Guatuso, UNA, Heredia, Costa Rica.

Secretaría de la Convención de Ramsar (2010), "Manejo de cuencas hidrográficas: integración de la conservación y del uso racional de los humedales en el manejo de las cuencas hidrográficas", en Manuales Ramsar para el uso racional de los humedales, Secretaría de la Convención de Ramsar, Gland, Suiza.

SINAC (Sistema Nacional de Áreas de Conservación) (2014a), "Parque Nacional Volcán Tenorio", Ministerio de Ambiente y Energía, San José, Costa Rica, <http://www.sinac.go.cr/ES/ac/acat/pnvt/ Paginas/default.aspx>, 8 de febrero de 2017. 
SINAC (Sistema Nacional de Áreas de Conservación) (2014b), "Refugio de Vida Silvestre Caño Negro", Ministerio de Ambiente y Energía, San José, Costa Rica, <http://www.sinac.go.cr/ES/ac/ACAHN/rnvscn/Paginas/default.aspx>, 8 de febrero de 2017.

Zumbado, Félix (2011), "Planes Reguladores y su importancia para el sector agropecuario. Realidades del ordenamiento territorial", Resista ECAG Informa, núm. 56, Escuela Centroamericana de Ganadería, Alajuela, Costa Rica, pp. 23-27.

Recibido: 14de abril de 2017. Corregido: 14 de julio de 2017. Aceptado: 14 de agosto de 2017.

Félix Zumbado-Morales. Master en Gestión ambiental y ecoturismo por la Universidad de Costa Rica, cursó la carrera de Ingeniería agronómica en la misma casa de estudios. Actualmente es investigador y coordinador de proyectos en el Programa de Investigación en Desarrollo Urbano Sostenible (ProDUS) de la Universidad de Costa Rica; es integrante de la Comisión de la Maestría en gestión ambiental y ecoturismo de la Universidad de Costa Rica así como de la Comisión de Manejo de Ecosistemas (CEM) de la Unión Internacional para la Conservación de la Naturaleza (UICN). Sus líneas de investigación son: Desarrollo rural, ordenamiento territorial, ecoturismo y gestión ambiental. Entre sus últimas publicaciones destacan: "Turismo rural y sector agropecuario en Costa Rica, perspectivas al año 2014”, Revista Iberoamericana de TurismoRITUR, 7 (1) Universidade Federal de Alagoas, Alagoas, Brasil, pp. 149171 (2017); "Planificación territorial y desarrollo rural en zonas de influencia de áreas naturales protegidas", Revista Espacio, Sociedady Turismo, 2 (4), Centro de Investigación en Geografía Aplicada, Lima Perú, pp. 45-56 (2015); en coautoría, Libro de texto para el curso de agroclimatología de la carrera de Agronomía, Universidad Estatal a Distancia UNED, San José, Costa Rica (2017).

Roger Mesén-Leal. Maestrando en Gestión de Recursos Naturales de la Universidad Estatal a Distancia de Costa Rica. Actualmente es investigador en el Programa de Investigación en Desarrollo Urbano Sostenible (ProDUS) de la Universidad de Costa Rica y profesor en la Universidad Latina de Costa Rica. Se ha especializado en temas de ordenamiento territorial y el uso de Sistemas de Información Geográfica como herramienta de análisis en investigaciones en temas de conservación de suelos, 
planificación territorial, cuencas hídricas y amenazas naturales. Entre sus últimas publicaciones destacan: Modificaciones al modelo de erosión, RUSLE para validar su aplicación en las cuencas de la Fila Costeña, durante las Jornadas de Investigación de la Facultad de Ingeniería, Universidad de Costa Rica, San José, Costa Rica (2010); Modificaciones en la Aplicación del modelo de erosión USLE-RUSLE para el ordenamiento territorial, en el V CICES, Universidad de Costa Rica, San José, Costa Rica (2010) y "Consideraciones y conclusiones al aplicar el modelo de erosión RUSLE en algunas cuencas de la fila costeña, Costa Rica”, XXIV Congreso Latinoamericano de Hidráulica, Universidad de la República, Punta del Este, Uruguay (2010). 